\title{
Measurement of dc Electrical Conductivity of Chemically Synthesized Pyrrole Aniline Copolymers
}

\author{
H R Wasnik ${ }^{1}$, D S Kelkar ${ }^{2}$, V Y Ganvir ${ }^{3}$ \\ ${ }^{I}$ (Department of Applied Physics, Yeshwantrao Chavan College of Engineering, Nagpur-440022, India.) \\ ${ }_{2}^{2}$ (Department of Physics, Institute of Science, Nagpur-440001, India.) \\ ${ }_{3}^{3}$ (Department of Applied Physics, Rajiv Gandhi College of Engineering \& Research, Nagpur-440022, India.)
}

\begin{abstract}
Chemical oxidative copolymerization of pyrrole and aniline was performed for three feed ratios at room temperature $\left(30^{\circ} \mathrm{C}\right)$ using $\mathrm{FeCl}_{3}$ as an initiator. The synthesis was carried out for two different initiator concentrations. The I-V plots for the respective samples have been plotted which indicate the linear or ohmic behavior of the samples with respect to field variation. The dc electrical conductivity of the copolymers was measured at a field of 27V/cm using two probe method in the temperature range of 313-673K. The log $\sigma$ versus 1/T graphs for these samples have been plotted in the same temperature range. The results show transitions of the samples from metallic to semiconducting behavior and vice versa depending upon each sample at a particular temperature specific for the sample. The results show reduction in the conductivity of prepared copolymers on comparison with that of the homopolymers.
\end{abstract}

Keywords: Aniline, copolymer, electrical conductivity, pyrrole.

\section{Introduction}

The usability of improved conducting materials with better performances for applications like thermal, electrical, electromagnetic shielding properties, display properties, [1-3] diodes, polymer batteries, photovoltaic cell, [4-6] etc. have received much attention nowadays. The designing of new microstructures via copolymerization serve as a tool for the synthesis of polyfunctional macromolecules with specific composition, properties, and structure thereby offering a technique to polymerize those monomers that do not polymerize independently [7].Thus a cross linked or hetero associated material with modified primarily existing polymeric chain can be designed and further employed to meet industrial and scientific requirements.

Among various conducting polymers polypyrrole and polyaniline are rigorously investigated due to their ease of preparation, environmental stability and high electrical conductivity. Despite of these advantages, it is observed that the two monomers are thermally quiet unstable. This puts restriction on the use of theses materials for higher temperature applications. However many researchers have employed electrochemical copolymerization technique for synthesizing a copolymer involving pyrrole aniline units [8-10].

Also people have studied the chemical copolymerization with equimolar proportion of the comonomers at lower temperatures ranging from $\left(0-5^{\circ} \mathrm{C}\right)[11-13]$. But these studies involve only room temperature or low temperature synthesis conditions. Again they have reported only room temperature conductivity of these prepared copolymers.

Thus in the present work, we report studies on chemical copolymerization of pyrrole and aniline for three different proportion of the comonomers. The polymerization was carried out at room temperature i.e. $\left(30^{\circ} \mathrm{C}\right)$ using $\mathrm{FeCl}_{3}$ as an initiator with two different concentrations. The I $-\mathrm{V}$ plots for all these copolymers have been plotted which shows ohmic characteristic owing to linearity. Further the dc electrical conductivity of these prepared materials was recorded using two probe method in the temperature range of $313-673 \mathrm{~K}$ at a field of $27 \mathrm{~V} / \mathrm{cm}$. The results show decrease in the conductivity of these copolymers when compared with that of homopolymers.

\subsection{Sample Preparation}

\section{Experimental}

Aniline (SRL Mumbai) and Pyrrole (SRL Mumbai) were purified by distillation process prior to use. Also sulphuric acid (Qualigen, Mumbai) was used to generate acidic medium and anhydrous $\mathrm{FeCl}_{3}$ (SRL Mumbai) was used as an initiator. In the present work the copolymerization has been carried out chemically by taking the monomers in the three different proportions. For this the two monomers were taken in desired proportion and the mixture was stirred for about 15 minutes. To it $30 \mathrm{ml}$ of $0.5 \mathrm{M} \mathrm{H}_{2} \mathrm{So}_{4}$ was added and the solution was stirred till it became clear. Finally measured quantity of anhydrous $\mathrm{FeCl}_{3}(0.1 \mathrm{M}$ and $0.25 \mathrm{M})$ was added to this solution and the whole reaction mixture was stirred for about 1 hour, continuously at a temperature of $30^{\circ} \mathrm{C}$. The reaction mixture was then allowed to polymerize for a period of $24 \mathrm{hrs}$. It was then filtered and the 
precipitate was washed with distilled water and acetone alternately till the filtrate became clear. The precipitate was then further dried and the weight of the material was recorded.

The nomenclature of the samples prepared with two different initiator concentrations has been given in table I.

\subsection{Measurement of de Electrical Conductivity}

The I - V characteristics for these copolymers have been plotted at a fixed temperature of $323 \mathrm{~K}$ by varying the field. The graphs have been plotted and are shown in fig I. Further the dc electrical conductivity measurement of the same sample was carried out with the help of two probe method. The conductivity was recorded within the temperature range $313 \mathrm{~K}$ to $673 \mathrm{~K}$ at a field of $27 \mathrm{~V} / \mathrm{cm}$. All the measurements were done with the help of KEITHLY 6514 system ELECTROMETER and Testronix digital regulated dc power supply (0 $-300 \mathrm{~V} \& 0-500 \mathrm{~mA})$ measuring instrument. The heating rate was maintained constant throughout the measurement i.e. $5^{\circ} \mathrm{C}$ per minute. The dc electrical conductivity was recorded by using the formula:

$$
\sigma=\frac{t}{A R}
$$

Where $\sigma=$ dc electrical conductivity

$\mathrm{A}=$ area of cross section of the palette

$\mathrm{R}=$ resistance of the sample

\section{Results And Discussion}

In order to ensure that during synthesis copolymer is formed and not mixtures of two homopolymers ( polyaniline and polypyrrole) solubility test was carried out. Polyaniline and polypyrrole are both completely insoluble in DMF ( Dimethyl Formamide) and DMSO ( Dimethyl Sulphoxide); whereas the synthesized material is soluble to a good extent in both of these solvents. Also the IR spectrum of the copolymers was compared with that of the physical mixtures of polypyrrole and polyanilne in 1:1 proportion (by weight). The bands obtained in both these cases are quite different from each other. Hence it can be said that copolymer has been formed.

Table I : Designation of samples, their percentage conversion at constant temperature \&room temperature conductivity.

\begin{tabular}{|c|c|c|c|c|}
\hline $\begin{array}{c}\text { Synthesis } \\
\text { Temperature }\end{array}$ & Initiator Concentration & Sample Designation & $\begin{array}{c}\text { Percentage Conversion } \\
\text { (Yield in \%) }\end{array}$ & $\begin{array}{c}\mathbf{\sigma} \\
\mathbf{( S / ~ c m )}\end{array}$ \\
\hline \multirow{5}{*}{$30^{\circ} \mathrm{C}$} & $0.1 \mathrm{M}$ & $\mathrm{X} 2$ & $15.9116 \%$ & $1.70105 \times 10^{-\mathbf{0 8}}$ \\
\cline { 2 - 5 } & $0.1 \mathrm{M}$ & $\mathrm{Y} 2$ & $6.1301 \%$ & $4.47711 \times 10^{-\mathbf{0 8}}$ \\
\cline { 2 - 5 } & $0.1 \mathrm{M}$ & $\mathrm{Z} 2$ & $28.9628 \%$ & $4.73312 \times 10^{-\mathbf{0 8}}$ \\
\cline { 2 - 5 } & $0.25 \mathrm{M}$ & $\mathrm{X} 5$ & $36.3988 \%$ & $7.94127 \times 10^{-\mathbf{0 8}}$ \\
\cline { 2 - 5 } & $0.25 \mathrm{M}$ & $\mathrm{Y} 5$ & $54.5236 \%$ & $4.51677 \times 10^{-\mathbf{0 8}}$ \\
\cline { 2 - 5 } & $0.25 \mathrm{M}$ & $\mathrm{Z}$ & $5.2592 \%$ & $1.18089 \times 10^{-\mathbf{0 7}}$ \\
\hline
\end{tabular}

Where $\mathrm{X}, \mathrm{Y} \& \mathrm{Z}$ represents the different proportions of the monomers indicated as :

\begin{tabular}{|c|c|}
\hline Designation & Pyrrole : Aniline \\
\hline $\mathrm{X}$ & $1: 1$ \\
\hline $\mathrm{Y}$ & $1: 2$ \\
\hline $\mathrm{Z}$ & $2: 1$ \\
\hline
\end{tabular}

The I-V plots for all the six copolymers have been shown in fig.I. Graph (a) \& (b) represents the variation of current through the sample for $0.1 \mathrm{M} \& 0.25 \mathrm{M}$ initiator concentration $\left(\mathrm{FeCl}_{3}\right)$ respectively. All the graphs are nearly linear indicating the ohmic nature of the sample. Thus from these graphs it can be observed that as voltage increases the current increases due to the alignment of the charge carriers in the direction of applied field.

The careful observation of both graphs shows that as the initiator concentration is increased from $0.1 \mathrm{M}$ to $0.25 \mathrm{M}$ the current values are reduced by three orders (i.e. from $\mu \mathrm{A}$ to $\mathrm{nA}$ ). This could be explained as: as the initiator concentration is increased more number of free radicals $\left(\mathrm{Fe}^{3+}\right.$ and $\mathrm{Cl}^{-1}$ are available in the reaction mixture causing more oxidation of the pyrrole monomer. But at the same time relative oxidation of aniline units is less hence they remain into the solution. This may cause the continuous collision of the unreacted aniline units and initiator units. These collisions in turn oppose the further reaction between the aniline and pyrrole monomers resulting in lowering of current values.

The $\log \sigma$ Vs $1 /$ T plots for all the copolymers are shown in figure II. Thus it is observed that every sample shows a transition from semi conducting to metallic behavior at a certain temperature and vice versa above this transition temperature. The room temperature dc electrical conductivity of copolymers is tabulated in 
table I. From table I it is observed that electrical conductivity has been reduced to a greater extent when compared to the parent homopolymers. This reduction in conductivity may be due to the nature of primary copolymer chain comprising of units of pyrrole and aniline. The charge carriers associated with these units can propagate only upto certain common sequences of either polymer. As soon as the hetero junction is achieved the energy of the charge carriers becomes insufficient for crossing the junction. Thus charge carriers get trapped or their motion is hindered due to the presence of second monomer unit. Hence the conjugation does not remain uniform over the entire polymer chain and overall reduction in the conductivity is observed. Further the steric effects arising due to the attachment of bulky substituents provide torsional twists in the copolymer backbone, reducing the co planarity and average electron delocalization length, and the conductivity [14-15].

Such type of observations has been noted for substituted polyaniline and copolymers of anilines with substituted anilines [16-17]. Hence copolymers so formed are not just the combinations of polypyrrole and polyaniline but a completely new material with new properties.

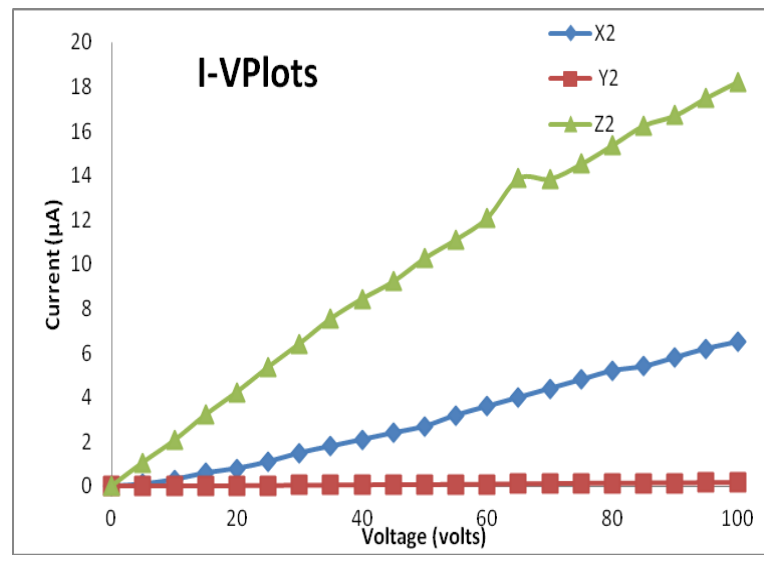

(a)

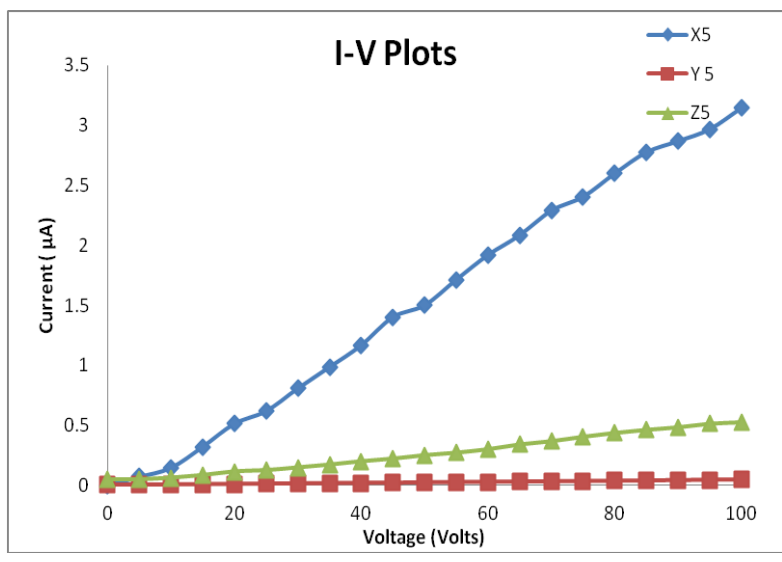

(b)

Fig.I : I-V plots for copolymers (a) Initiator concentration $=0.1 \mathrm{M}$ (b) Initiator concentration $=0.25 \mathrm{M}$

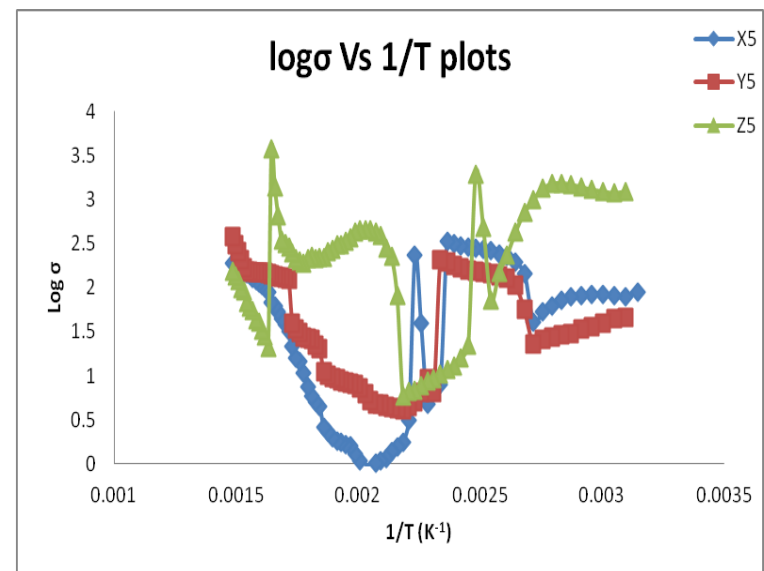

(a)

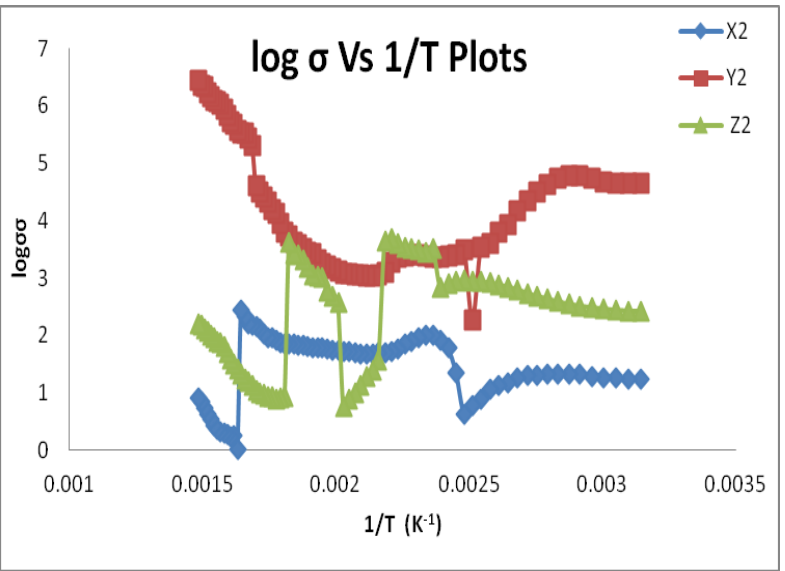

(b)

Fig.II : $\log \sigma$ versus $1 / \mathrm{T}$ plots for copolymers (a) Initiator concentration $=0.1 \mathrm{M}$ (b) Initiator concentration $=\mathbf{0 . 2 5 M}$

\section{Conclusion}

Copolymers of pyrrole and aniline have been synthesized by using chemical oxidative copolymerization method. All the copolymers have been synthesized at room temperature with different feed ration and initiator concentrations. Further dc electrical conductivity of these copolymers has been measured by using two probe method at constant temperature $(323 \mathrm{~K})$ and at consatant field $(27 \mathrm{~V} / \mathrm{cm})$. Graphs showing variation of conductivity with temperature as well as I-V plots have been plotted and studied. These graphs show that conductivity reduces to a large extent for all copolymers when compared to parent homopolymers. 


\section{References}

[1]. A. Kaynak , Electromagnetic shielding effectiveness of galvanostatically synthesized conducting polypyrrole films in the 300-2000 MHz frequency range, Materials Research Bulletin, 31(7) ,1996, 845 - 860.

[2]. J. Iroh, K. Levine, K. Shah, Y. Zhu, M. Donley, R. Mantz, J. Johnson, N. Voevodin, V. Balbyshev, A. Khramov, Electrochemical Behaviour of Conducting Polymer/Polyimide Composite, Surface Engineering, (20), 2004 , 93 - 98.

[3]. T. Makela, S. Pienimaa, T. Taka, S. Jusslia, H. Isotalo, Thin polyaniline films in EMI shielding, Synthetic Metals 85 (1-3), 1997, 1335-1336.

[4]. S. Panero, P. Prosperi, F. Bonono, B. Scrosati, A. Corradini, M. Mastragostino, Characteristics of electrochemically synthesized polymer electrodes in lithium cells-III. Polypyrrole, Electrochimica Acta, 32 (7), 1987, 1007 -1011.

[5]. T. Otero, I. Cantero, B. Azcano, Optimization of poly-3-methylthiophene to be used in advance polymer batteries, International Journal of Hydrogen Energy 25, 2000, $221-233$,

[6]. A. MacDiarmid, L. Yang, W. Huang, B. Humphrey, Polyaniline: Electrochemistry and application to rechargeable batteries, Synthetic Metals , 18, 1987, $393-398$.

[7]. C Yang, P. Smith, A. Heeger, Y. Cao, Electron diffraction studies of the structure of polyaniline dodecylbenzenesulfonate , Polymer, 35, 1994, 1142-1144.

[8]. O Inganas, B. Liedberg, Wu Chang Ru, A new route to polythiophene and copolymers of thiophene and pyrrole, Synthetic Metals , 11(4-5), $1985,239-249$.

[9]. Y. Sahin, S. Percine, M. Sahin, G. Ozkan G, Electrochemical polymerization of fluoro \& chloro substituted anilines \& Copolymers with aniline, Journal of Applied Polymer Science, 91, 2004, 2302-2312.

[10]. D. Borole, U. Kapadi, P. Mahulikar, D. Hundiwale, Electrochemical synthesis and characterization of conducting copolymer: Poly(o-aniline-co-o-toluidine), Material Letters, 60 , 2006, 2447-2452.

[11]. A. Jenkins, Advances in free radical chemistry-volume II ( Academic Press, 1986)

[12]. V. W. L. Lim, E. Kang, K. Neoh, Z. Ma, K. Tan, Determination of pyrrole-aniline copolymer compositions by X-ray photoelectron spectroscopy, Applied Surface Science, $181,2001,317-326$.

[13]. Q. Liang, K. Neoh, E. Kang, K. Tan, H. Wong, Charge transfer interactions in Bithiophene-pyrrole and Bithiophene-aniline copolymer, European . Polymer Journal, 28 (7), 1992, 755-763.

[14]. L. Zhou , G. Xue, Electrochemical synthesis of free standing poly(3-bromothiophene) polymer , Synthetic Metal, 87 (3) ,1997,193-195

[15]. M. Lemaire , W. Buchner, R. Garreau , H. Hoa , A. Guy, J. Roncali , Synthesis of organic conductors using electrodesilylation , Journal of Electroanalytical Chemistry and Interfacial electrochemistry ,281(1-2), 1990, 293-298.

[16]. S. Chen, G. Huang, Water soluble self acid doped conducting polyaniline : structure and properties, Journal of American Chemical society, 117(40), 1995, 10055-10062.

[17]. L. Huang, T. .Wen, A. Gopalan, Synthesis and characterization of soluble conducting poly(aniline -co-2,5-dimethoxyaniline , Materials Letters. 57, 2003 1765-1774. 